\title{
Suicide among cancer patients: adolescents and young adult (AYA) versus all-age patients
}

\author{
Huaqiang Zhou ${ }^{1,2,3,4}$, Wei Xian ${ }^{4 \#}$, Yaxiong Zhang ${ }^{1,2,3 \#}$, Yunpeng Yang ${ }^{1,2,3}$, Wenfeng Fang ${ }^{1,2,3}$, Jiaqing Liu ${ }^{4}$, \\ Jiayi Shen ${ }^{4}$, Zhonghan Zhang ${ }^{1,2,3}$, Shaodong Hong ${ }^{1,2,3}$, Yan Huang ${ }^{1,2,3}$, Li Zhang ${ }^{1,2,3}$ \\ ${ }^{1}$ Department of Medical Oncology, Sun Yat-sen University Cancer Center, Guangzhou 510060, China; ${ }^{2}$ State Key Laboratory of Oncology in South \\ China, Guangzhou 510060, China; ${ }^{3}$ Collaborative Innovation Center for Cancer Medicine, Guangzhou 510060, China; ${ }^{4}$ Zhongshan School of \\ Medicine, Sun Yat-sen University, Guangzhou 510080, China \\ Contributions: (I) Conception and design: L Zhang, H Zhou, W Xian, Y Zhang; (II) Administrative support: None; (III) Provision of study materials \\ or patients: None; (IV) Collection and assembly of data: None; (V) Data analysis and interpretation: All authors; (VI) Manuscript writing: All authors; \\ (VII) Final approval of manuscript: All authors. \\ \#These authors contributed equally to this work. \\ Correspondence to: Li Zhang. Department of Medical Oncology, Sun Yat-sen University Cancer Center, 651 Dongfeng Road East, Guangzhou \\ 510060, China. Email: zhangli6@mail.sysu.edu.cn.
}

\begin{abstract}
Background: Many researchers have studied suicide risk factors of patients with one specific cancer. But there is no comprehensive study to compare suicide issues between adolescents and young adult (AYA) group and all-age groups in a pan-cancer view.

Methods: Patients diagnosed with 20 solid malignancies were identified from SEER database. Multivariable logistic regression was operated to find out risk factors of suicide.

Results: Male sex has less impact on AYA than all-age patients (OR 2.72, 95\% CI: 2.23-3.31, $\mathrm{P}<0.001$ vs. OR 4.64, 95\% CI: 4.37-4.94, $\mathrm{P}<0.001$ ), while white race (OR 3.28, 95\% CI: 2.02-5.77, $\mathrm{P}<0.001$ vs. OR 3.40, 95\% CI: 3.02-3.84, $\mathrm{P}<0.001$ ) and unmarried status (OR 1.51, 95\% CI: 1.24-1.83, P<0.001 vs. OR 1.39, 95\% CI: $1.33-1.46, \mathrm{P}<0.001)$ have similar impact on AYA and all-age groups. Localized cancer stage may have stronger impact on AYA than all-age (OR 2.90, 95\% CI: 1.83-4.84; $\mathrm{P}<0.001$ vs. OR 1.76, 95\% CI: 1.61-1.92; $\mathrm{P}<0.001)$, while surgery only influence all-age (OR $1.14, \mathrm{P}=0.451$ vs. $1.24, \mathrm{P}<0.001)$. Within 5 years from cancer diagnosis, longer survival time is associated with higher suicide risk of both all-age and AYA patients.

Conclusions: Male sex, white race and unmarried status, earlier cancer stage and longer survival time within 5 years are similar prevalent risk factors for both AYA group patients and all-age patients. It is not necessary to pick AYA cancer patients out when considering suicide risk of cancer patients.
\end{abstract}

Keywords: Suicide; adolescents and young adult (AYA); Surveillance, Epidemiology, and End Results (SEER); risk factors; prevention

Submitted Jun 23, 2019. Accepted for publication Sep 26, 2019.

doi: 10.21037/atm.2019.10.51

View this article at: http://dx.doi.org/10.21037/atm.2019.10.51

\section{Introduction}

Suicide accounted for $1.4 \%$ of all deaths worldwide, making it one of leading cause of death in 2016 (1). Cancer patients in US have nearly twice the suicide rate of the general population (2). Researchers have demonstrated suicide risk factors of patients with one specific cancer, such as lung cancer, colorectal cancer, head and neck cancer and so on (3-7). It has been discovered that male sex, white race, and older age at diagnosis may be associated with higher risk of suicide among cancer patients (2).

Adolescents and young adults (AYA) are defined as people whose age is between 15 and 39 (8). In 2017, adolescents and young adults aged 15 to 24,25 to 34,35 to 44 had a 
suicide rate of $14.46,17.53,17.94$ per 100,000 individuals in US respectively (9). About 70,000 adolescence and young adult (ages 15-39) are diagnosed with cancer each year in the United States, and cancer is the leading cause of diseaserelated death in the AYA population (8). Additionally, cancer incidence increased faster in AYAs than in younger or older population (10). Nowadays, AYA cancer patients lose advantage of prognosis compared to older cancer patients, and survival quality of AYA cancer patients should be focused on (11).

However, there is no comprehensive study to compare suicide issues between AYA group and all-age group in pancancer view. The purpose of this research is to analyze the necessity to separate AYA cancer patients as a special group to identify high risk patients.

\section{Methods}

Eligible cancer survivors were identified from National Cancer Institute's Surveillance, Epidemiology, and End Results (SEER) database. SEER is a publicly accessible source of cancer patient information in the United States. This database collects cancer incidence data from population-based cancer registries covering approximately 34.6 percent of the U.S. population diagnosed between 1973 and 2015 (12).

Data of patients diagnosed with 20 types of solid malignancies (tumors of the nasopharynx, esophagus, stomach, colon and rectum, liver, gallbladder, pancreas, lung and bronchus, bones, joints and soft tissue, melanoma, breast, cervix uteri, corpus and uterus, ovary, prostate, urinary bladder, kidney and renal pelvis, eye and orbit, brain and other nervous system, and thyroid) were obtained from SEER database (1973-2015) using the SEER*Stat software (v8.3.5, Cancer Statistic Branch, NCI, Calverton), using International Classification of Disease for Oncology, Third Edition (ICD-O-3), morphology codes (13). Patients with unknown follow-up or recorded by autopsy and death certificate were eliminated.

Inclusion of patients with multiple primary cancers may have potential effect on suicidal behaviors which may lead to different result of high-risk population $(5,14)$. To prevent the interference of multiple primary cancers, we extracted data of patients diagnosed with only one primary cancer of 20 kinds of solid carcinoma. Demographic and clinicopathological variables of interest encompassed sex, race, marital status, age at diagnosis, year of diagnosis, grade, stage, surgery, cause of death and survival time.
Patients whose cause of death variable coded as "Suicide and self-inflicted injury" were identified. They were divided into 3 groups according to age at diagnosis (younger than 15 years, 15-39 years, and older than 40 years). We sorted patients by white, black and others, and categorized patients as married or unmarried. Year of diagnosis were allocated into 3 groups (before 1993, 1994-2003, after 2004). Surgery was classified as performed, not performed, and unknown. Disease stage for the analysis was coded based on the variable "SEER Historic Stage A". We give a value of 0.5 months to those who didn't survive for a full month after diagnosis, because SEER record their survival time in months (13).

To examine patients committed suicide with those died from other causes, univariate analysis using chi-square test was applied. We employed multivariable logistic regression to distinguish independent risk factors for suicide. All statistical analyses were performed using $\mathrm{R}$ version 3.4.2 software (Institute for Statistics and Mathematics, Vienna, Austria; www.r-project.org). Statistical significance was set at two-sided $\mathrm{P}<0.05$.

\section{Results}

\section{Patient cobort characteristics}

We identified 5,261,459 patients, and 312,313 of them are AYA patients. The SMR for AYA is slightly lower than that for all identified patients $(0.14 \%, 433$ in 312,313 vs. $0.15 \%$, 7,831 in $5,261,459, \mathrm{P}=0.150$ ) (Table S1). Highest SMR is seen in nasopharynx cancer $(0.34 \%$ vs. $0.38 \%)$, prostate cancer $(0.26 \%$ vs. $0.29 \%)$ and urinary bladder cancer $(0.26 \%$ vs. $0.37 \%)$ when comparing AYA and all-age patients. Decrease of SMR is observed both in AYA group and allage group cancer patients as time goes by (Tables $S 2, S 3)$.

\section{Risk factors of suicide in AYA group and all-age group cancer survivors}

When analyzed in pan-cancer view, male sex, white race, unmarried status, earlier stage and later time from cancer diagnosis are suicide risk factors for both AYA and all-age cancer patients. The effect of gender on the AYA group was not as obvious as that of the whole age group when consider all cancers (OR 2.72, 95\% CI: 2.23-3.31, $\mathrm{P}<0.001$ vs. OR 4.64, 95\% CI: 4.37-4.94, $\mathrm{P}<0.001$ ) (Table 1). Race has similar effects on AYA patients and all-age patients (OR $3.28,95 \%$ CI: $2.02-5.77, \mathrm{P}<0.001$ vs. OR $3.40,95 \%$ CI: 


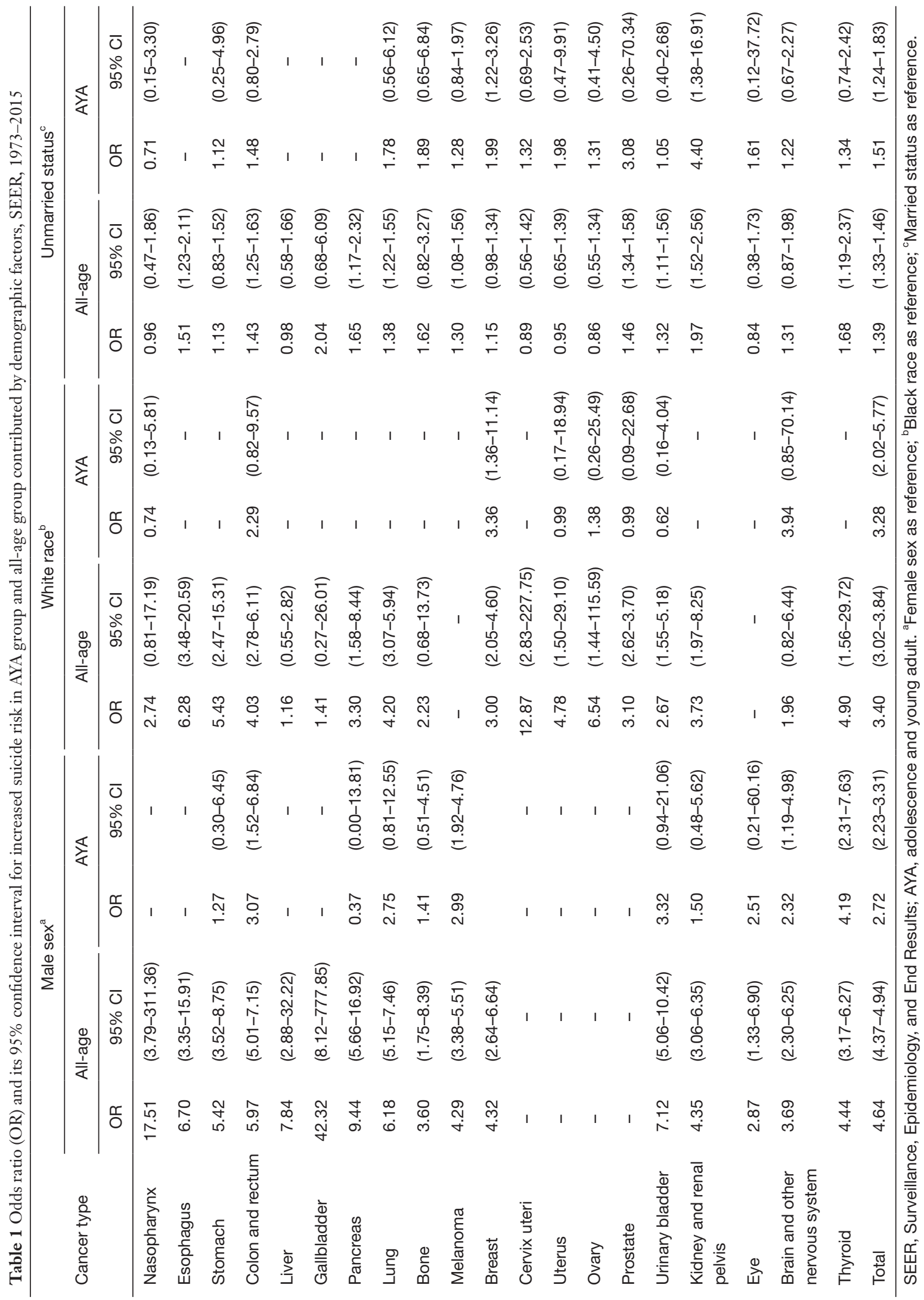




\section{Page 4 of 10}

3.02-3.84, $\mathrm{P}<0.001$ ) (Table 1). Impact of marital status on AYA patients and all-age patients are similar as well (OR 1.51, 95\% CI: $1.24-1.83, \mathrm{P}<0.001$ vs. OR 1.39, 95\% CI: $1.33-1.46, \mathrm{P}<0.001$ ) (Table 1). Localized stage of cancer is associated with higher risk of suicide in all-age patients, and the association is stronger in AYA patients (OR 1.76, 95\% CI: $1.61-1.92, \mathrm{P}<0.001$ vs. OR 2.90, 95\% CI: 1.83-4.84, $\mathrm{P}<0.001$ ) (Table 2) Surgery is related to suicide in allage cancer patients, but it don't have significant impact on suicide ideal in AYA cancer patients (OR 1.24, 95\% CI: $1.17-1.31, \mathrm{P}<0.001$ vs. OR 1.14, 95\% CI: 0.82-1.59, $\mathrm{P}=0.451$ ) (Table 2). Within 5 years from cancer diagnosis, longer survival time is associated with higher suicide risk of both all-age and AYA patients (Table 3).

Suicide risk of specific cancer patients in AYA group and all-age group is affected by the same demographic factor differently. Compared to female patients, male patients with pancreas cancer are most likely to suicide in all ages (OR 9.44, 95\% CI: 5.66-16.92, $\mathrm{P}<0.001$ ) (Table 1), while male AYA patients with thyroid cancer have highest risk of suicide (OR 4.19, 95\% CI: 2.31-7.63, $\mathrm{P}<0.001$ ) (Table 1). When considering ethnicity of patients, white patients with esophagus cancer take higher risk of suicide (OR 6.28, 95\% CI: 3.48-20.59, $\mathrm{P}<0.001$ ) (Table 1) while white AYA patients with breast cancer (OR 3.36, 95\% CI: 1.36-11.14, $\mathrm{P}=0.020$ ) (Table 1) are most potential to commit suicide. As for unmarried patients, those with kidney and renal pelvis cancer are most likely to suicide both in AYA (OR 4.40, 95\% CI: 1.38-16.91, $\mathrm{P}=0.017$ ) (Table 1) and in all (OR 1.97, 95\% CI: 1.52-2.56, $\mathrm{P}<0.001$ ) (Table 1).

Clinicopathological factor also have different influence on suicide risk of specific cancer patients in AYA group and all-age group. When using distant stage as reference, localized stage has greatest impact on breast cancer patients both in all-age group (OR 5.16, 95\% CI: 3.17-8.96, $\mathrm{P}<0.001$ ) and AYA group (OR 7.55, 95\% CI: 1.47-139.76, $\mathrm{P}=0.055$ ) (Table 2). Compared to those didn't undergo surgery, brain and other nervous system cancer patients who undergo surgery have higher risk of suicide (OR 1.95, 95\% CI: $1.21-3.31, \mathrm{P}=0.009$ ) (Table 2), and surgery don't have significant impact on specific type of cancer in AYA patients as well.

Suicide risk of specific cancer patients in AYA group and all-age group influenced by period from cancer diagnosis differently. At the first year of cancer diagnosis, all-age patients with breast cancer are most likely to suicide (OR 3.71, 95\% CI: 2.92-4.67, $\mathrm{P}<0.001$ ). While AYA patients with cervical cancer have highest
Zhou et al. Suicide risk among AYA and all-age cancer survivors

suicide risk (OR 5.11, 95\% CI: 1.98-12.24, $\mathrm{P}<0.001$ ) (Table 3). Ovarian cancer patients are most likely to suicide in all-age group (OR 2.65, 95\% CI: 1.03-6.07, P=0.029) and kidney and renal pelvis cancer patients have highest suicide risk in AYA group (OR 15.22, 95\% CI: 2.97-62.34, $\mathrm{P}<0.001)$ at the fifth year of cancer diagnosis.

\section{Discussion}

Our study observed that suicide rate of AYA cancer patients is marginally lower than all-age cancer patients. On one hand, AYA cancer patients may use internet more frequently than older patients or children $(15,16)$. They may acquire more information about their cancer and psychological support from the Internet, which may enhance their confidence to fight against cancer and lower their ideal of suicide $(15,17)$. On the other hand, AYA cancer patients may have need of fertility preservation, and it may provide AYA cancer patients the impetus to survive (18). According to the result, both AYA patients and all-age patients with nasopharynx cancer, prostate cancer and urinary bladder cancer have highest SMR. But former researches showed highest suicide rate occur in lung, stomach, and head and neck cancers patients, which is not completely consistent with our result (2). Also, the sites correlated with the greatest suicide rate vary depending on the report $(3,19,20)$. The underlying reason is unknown, and it may be explained that nasopharynx cancer has a catastrophic impact on patients' quality of life $(21,22)$. Contrary to increasing suicide rate of US population, significant improvement in suicide prevention is observed both in AYA group and all-age group cancer patients (23). This result is consistent with previous studies $(20,24)$. It may be explained by the improvement of prognosis of all-age cancer patients and AYA cancer patients due to development of advanced therapy such as targeted chemotherapy and immunotherapy $(10,25-28)$.

\section{Clinical implications}

Demographic factors of patients show close relationship with suicide risk of both AYA cancer patients. We discovered that male patients of both AYA group and all-age group patients are more likely to commit suicide, which is accordant with US general population (1). Previous studies also displayed that male sex is risk factor of suicide in many types of cancer patients $(2,29)$. However, male sex has less impact on suicide risk of AYA cancer patients compared 
Table 2 Odds ratio (OR) and its $95 \%$ confidence interval for increased suicide risk in AYA group and all-age group contributed by clinicopathological factors, SEER, 1973-2015

\begin{tabular}{|c|c|c|c|c|c|c|c|c|c|}
\hline \multirow{2}{*}{ Cancer type } & \multicolumn{5}{|c|}{ Tumor stage $^{a}$} & \multicolumn{4}{|c|}{ Surgery $^{b}$} \\
\hline & Stage & OR & $95 \% \mathrm{Cl}$ & OR & $95 \% \mathrm{Cl}$ & OR & $95 \% \mathrm{Cl}$ & OR & $95 \% \mathrm{Cl}$ \\
\hline \multirow[t]{2}{*}{ Nasopharynx } & Localized & 3.37 & (0.63-25.03) & - & - & 0.99 & $(0.39-2.16)$ & 0.54 & $(0.03-3.48)$ \\
\hline & Regional & 2.88 & $(0.81-18.42)$ & 1.66 & $(0.23-35.09)$ & & & & \\
\hline Esophagus & Regional & 1.73 & $(1.47-1.96)$ & - & - & & & & \\
\hline \multirow[t]{2}{*}{ Stomach } & Localized & 1.62 & $(1.04-2.49)$ & 2.53 & $(0.24-21.81)$ & 1.04 & $(0.73-1.48)$ & 1.44 & $(0.23-8.06)$ \\
\hline & Regional & 1.04 & $(0.68-1.59)$ & 0.73 & $(0.03-7.49)$ & & & & \\
\hline Colon and rectum & Localized & 2.34 & $(1.87-2.95)$ & 4.25 & $(1.36-16.21)$ & 0.90 & $(0.72-1.15)$ & 1.03 & $(0.36-3.47)$ \\
\hline Liver & Regional & 0.92 & $(0.38-2.27)$ & - & - & & & & \\
\hline \multirow[t]{2}{*}{ Gallbladder } & Localized & 0.54 & $(0.09-2.67)$ & - & - & 2.00 & $(0.57-8.09)$ & - & - \\
\hline & Regional & 1.00 & $(0.27-3.51)$ & - & - & & & & \\
\hline \multirow[t]{2}{*}{ Pancreas } & Localized & 0.99 & $(0.50-1.79)$ & 12.22 & $(0.27-691.59)$ & 1.08 & $(0.62-1.80)$ & 0.74 & $(0.01-40.38)$ \\
\hline & Regional & 0.89 & $(0.56-1.36)$ & - & - & & & & \\
\hline \multirow[t]{2}{*}{ Lung } & Localized & 1.33 & $(1.08-1.64)$ & 0.41 & $(0.02-5.51)$ & 1.47 & $(1.26-1.71)$ & 2.40 & $(0.57-11.29)$ \\
\hline & Regional & 1.18 & $(0.99-1.40)$ & 1.63 & $(0.22-14.54)$ & & & & \\
\hline Bone & Localized & 4.28 & $(1.49-14.40)$ & 3.41 & (0.62-64.54) & 1.25 & $(0.60-2.70)$ & 0.95 & $(0.29-3.67)$ \\
\hline \multirow[t]{2}{*}{ Cervix uteri } & Localized & 4.05 & $(1.32-17.74)$ & 6.95 & $(1.29-129.59)$ & 1.05 & $(0.58-1.94)$ & 0.49 & $(0.23-1.11)$ \\
\hline & Regional & 1.74 & $(0.56-7.61)$ & 2.07 & $(0.34-39.38)$ & & & & \\
\hline \multirow[t]{2}{*}{ Uterus } & Localized & 2.62 & $(1.17-7.01)$ & - & - & 1.62 & $(0.78-3.78)$ & - & - \\
\hline & Regional & 2.83 & $(1.18-7.87)$ & - & - & & & & \\
\hline \multirow[t]{2}{*}{ Ovary } & Localized & 2.30 & $(1.28-4.08)$ & 3.56 & $(0.81-26.16)$ & 0.78 & $(0.41-1.54)$ & 0.37 & $(0.04-8.72)$ \\
\hline & Regional & 1.70 & $(0.69-3.60)$ & - & - & & & & \\
\hline Prostate & $\begin{array}{l}\text { Localized/ } \\
\text { regional }\end{array}$ & 1.49 & $(1.19-1.89)$ & - & - & 1.05 & $(0.97-1.14)$ & 1.31 & $(0.10-34.88)$ \\
\hline \multirow[t]{2}{*}{ Urinary bladder } & Localized & 4.40 & $(2.21-10.41)$ & - & - & 1.17 & $(0.81-1.76)$ & - & - \\
\hline & Regional & 4.39 & $(2.19-10.42)$ & - & - & & & & \\
\hline
\end{tabular}

Table 2 (continued) 
Table 2 (continued)

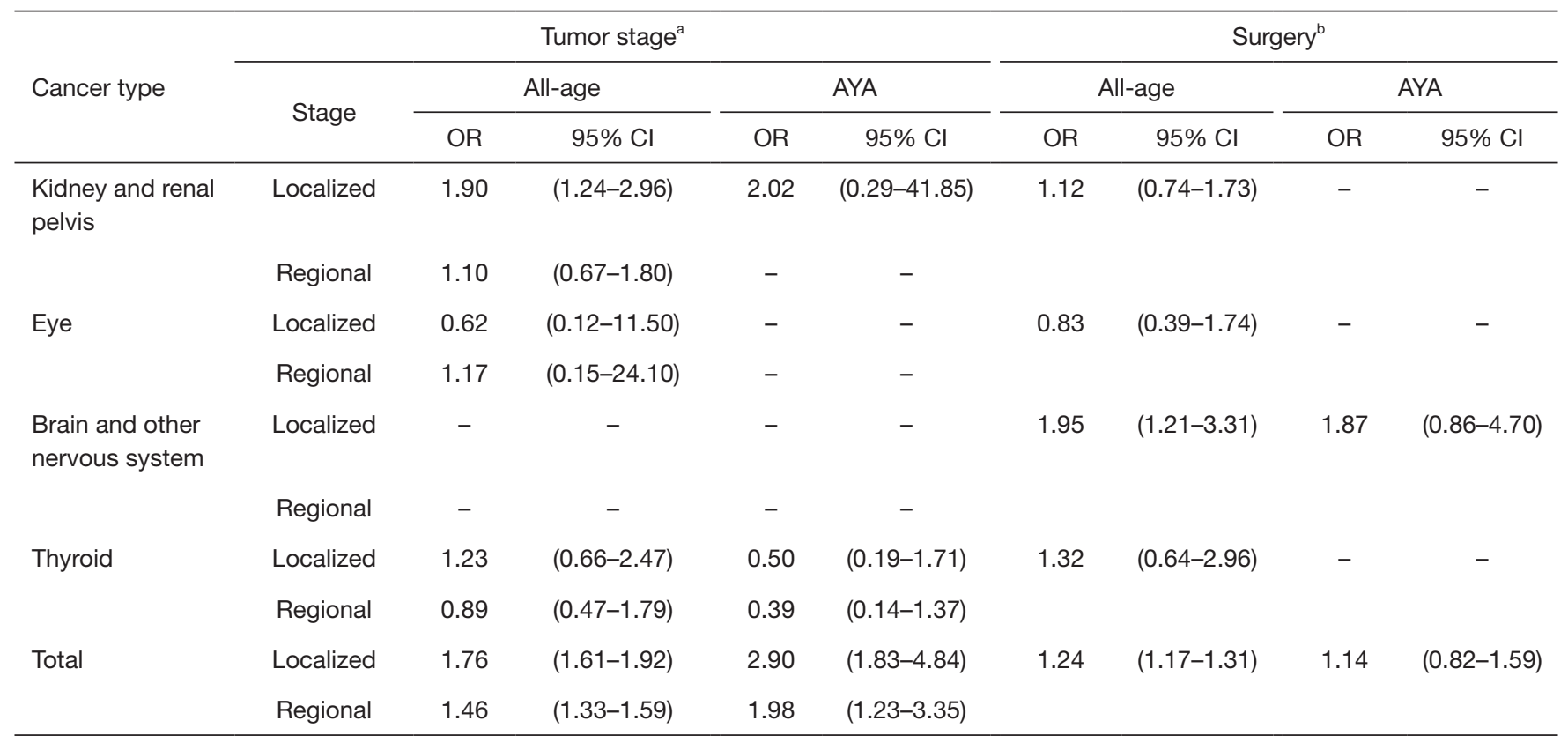

SEER, Surveillance, Epidemiology, and End Results; AYA, adolescence and young adult. ${ }^{a}$ Distant tumor stage as reference; ${ }^{b}$ Not undergoing surgery as reference.

Table 3 Odds ratio (OR) and its 95\% confidence interval for increased suicide risk in AYA group and all-age group contributed by time from cancer diagnosis, SEER, 1973-2015

\begin{tabular}{|c|c|c|c|c|c|c|c|c|c|c|}
\hline Cancer type & \multicolumn{2}{|c|}{1 year $^{a}$} & \multicolumn{2}{|c|}{2 years $^{a}$} & \multicolumn{2}{|c|}{3 years $^{a}$} & \multicolumn{2}{|c|}{4 years $^{a}$} & \multicolumn{2}{|c|}{5 years $^{a}$} \\
\hline \multicolumn{11}{|l|}{ All-age } \\
\hline Nasopharynx & 1.23 & $(0.56-2.67)$ & 0.66 & $(0.19-1.79)$ & 0.25 & $(0.01-1.24)$ & 1.77 & $(0.58-4.56)$ & - & - \\
\hline Esophagus & 1.75 & $(0.87-4.06)$ & 0.98 & $(0.58-2.48)$ & 1.10 & $(0.38-2.03)$ & 0.90 & $(0.38-3.14)$ & 2.96 & $(0.97-8.55)$ \\
\hline Colon and rectum & 1.25 & $(1.03-1.51)$ & 1.56 & $(1.27-1.91)$ & 1.65 & $(1.32-2.05)$ & 1.33 & $(1.01-1.71)$ & 1.46 & $(1.10-1.91)$ \\
\hline Liver & 1.46 & $(0.48-6.39)$ & 3.05 & $(0.96-13.52)$ & 1.74 & $(0.38-8.98)$ & 1.47 & $(0.19-8.98)$ & 3.25 & $(0.60-17.73)$ \\
\hline Gallbladder & 0.81 & $(0.12-16.47)$ & - & - & 2.46 & $(0.22-54.73)$ & - & - & - & - \\
\hline Pancreas & 1.27 & $(0.45-5.32)$ & 1.39 & $(0.45-6.03)$ & 2.17 & $(0.62-9.95)$ & 1.17 & $(0.15-7.12)$ & - & - \\
\hline Breast & 3.71 & $(2.92-4.67)$ & 2.77 & $(2.14-3.54)$ & 2.55 & (1.95-3.29) & 2.03 & $(1.49-2.70)$ & 2.04 & $(1.49-2.75)$ \\
\hline Cervix uteri & 2.20 & $(1.05-4.40)$ & 2.38 & $(1.07-4.87)$ & 1.45 & $(0.43-3.73)$ & 1.50 & $(0.36-4.26)$ & 2.43 & $(0.71-6.25)$ \\
\hline Uterus & 3.17 & $(1.80-5.42)$ & 2.04 & $(1.17-3.87)$ & 2.06 & $(0.93-4.05)$ & 0.55 & $(0.09-1.80)$ & 2.56 & $(1.11-5.19)$ \\
\hline Ovary & 1.40 & $(0.69-2.80)$ & 1.72 & $(0.80-3.57)$ & 1.65 & $(0.67-3.70)$ & 2.28 & $(0.92-5.09)$ & 2.65 & $(1.03-6.07)$ \\
\hline
\end{tabular}

Table 3 (continued) 
Table 3 (continued)

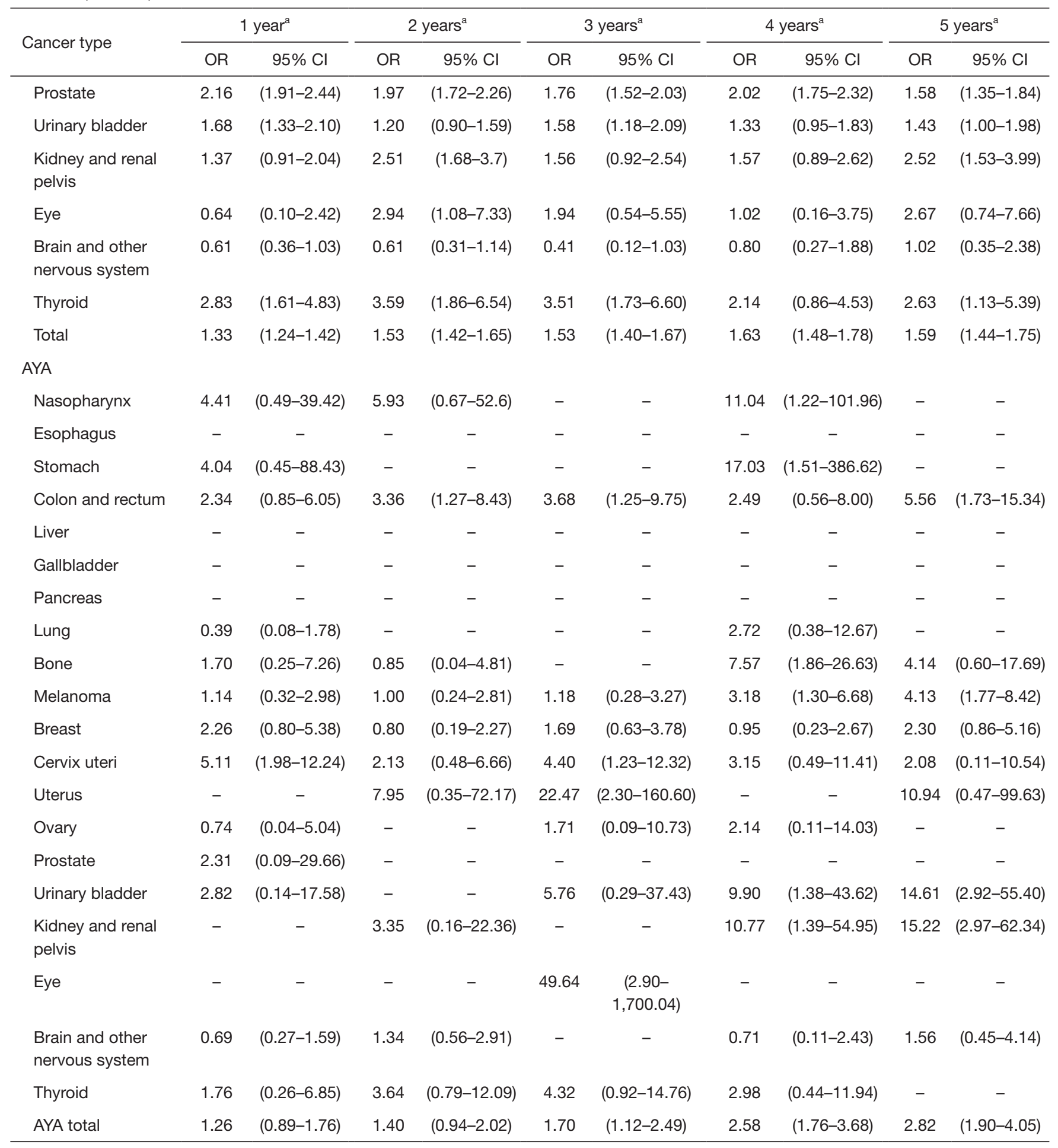

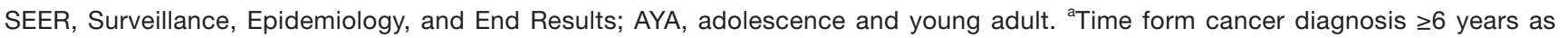
reference. 
to all-age patients. AYA cancer patients may suffer from harder financial situation than other patients, and male patients may be the breadwinner of his family (30). Thus, male AYA patients would rather struggle to live than ending their live. Another possible reason is that female AYA patients may have higher suicidal thoughts than other age patients (31). White patients also have higher risk of committing suicide, and race has a similar impact on suicidal ideation of AYA and all-age group. Former research discovered that black and white did not significantly differ in frequency of psychiatric conditions in suicide patients in Michigan HealthCare System (32). The reason for higher suicide risk in white patients in all-age group and AYA group remains unknown, and hopelessness may be associated with higher suicide risk in white patients (33). Unmarried status is also relative with greater suicide risk of AYA and all-age cancer patients. Impact of marital status on suicide risk of AYA group and all-age group is alike. A recent review has shown that both objective social isolation and the subjective feeling of loneliness due to unmarried status can explain higher suicide risk in AYA and older patients (34).

Interestingly, when analyze suicide risk in specific cancer patients, male sex and white race and marital status have different effect on all-age group and AYA group. The underlying reason is unknown, and the possible reason is that different cancer may have different impact on life of quality and mental health of all-age patient and AYA patients $(21,35,36)$. Also, AYA patients with different cancer may undergo different survivorship care patterns compared to cancer patients in other age (37).

When considering clinicopathological factors, localized tumor is related to higher risk of suicide in all-age cancer patients and AYA cancer patients. It has been reported that early stage is suicide risk factor of penile cancer patients (6). However, previous studies showed advanced stage cancer patients tend to suicide, which is different with our study $(2,3,38)$. A possible reason is that patients with distant stage may die from the disease itself rather than suicide because of the poor prognosis of advanced cancer. Surgery is related to suicide in all-age cancer patients, but it doesn't have significant impact on suicide ideal in AYA cancer patients. Curative surgery may result in weakness and loss of autonomy in a population in older people, and AYA group patients may have stronger resistance to weakness caused by surgery (39).

Longer period from cancer diagnosis is associated with higher suicide risk of both all-age patient and AYA patients within 5 years from diagnosis. Previous study showed Austrian cancer patients is most likely to commit suicide within 1 year from diagnosis, which is different from our result (40). The different result may be explained by accumulated pain in treatment and poor quality of life in long-term survivors of cancer (41). When analyzed in specific cancer, breast cancer patients in all-age group have highest suicide risk in first year from cancer diagnosis, which is consistent with former research (7). In AYA group, cervical cancer patients have highest suicide risk in first year from cancer diagnosis. A possible reason is that cervical cancer may deprive fertility of AYA patients, leading to depression of AYA patients (42).

Besides demographic and clinicopathological factors mentioned above, suicidal behavior may also be influenced by personal and psychological factors $(43,44)$. A prospective study reported that early onset of tobacco use increase risk of suicidal behaviors in AYA patients significantly (45). Use of alcohol and marijuana may also predict higher risk of suicide in AYA patients (46). Lower education level and unemployment caused by cancer may also associated with higher risk of suicide in AYA patients $(44,47)$. In addition, infertility increased risk of suicidal attempts of AYA patients so it is important to preserve fertility of AYA patients during treatment of cancer (48).

\section{Study limitations}

Our study still has some limitations. SEER is a database only contains information of US cancer patients, so our study has a weakness of research area, and research over the world is still needed. Suicide is a complicated phenomenon affected by many factors, but detailed information such as socioeconomic status and treatment details is not available in SEER database.

\section{Conclusions}

Male sex, white race, unmarried status, localized cancer stage and longer survival time within first 5 year of cancer diagnosis are prevalent risk factors for both AYA group patients and all-age patients, and risk factors of suicide have similar effects on patients in different age groups. Therefore, it is unnecessary to separate AYA patients as a specific group to identify patients with higher suicide risk.

\section{Acknowledgments}

Funding: This work was supported by the National Key 
R\&D Program of China (Grant No. 2016YFC0905500, 2016YFC0905503), Science and Technology Program of Guangdong (Grant No. 2017B020227001), Science and Technology Program of Guangzhou (Grant No. 201607020031), Chinese National Natural Science Foundation Project (Grant No. 81772476, 81572659, 81602005), Pearl River Nova Program of Guangzhou (Grant No. 201610010048).

\section{Footnote}

Conflicts of Interest: The authors have no conflicts of interest to declare.

Ethical Statement: The authors are accountable for all aspects of the work in ensuring that questions related to the accuracy or integrity of any part of the work are appropriately investigated and resolved. Approval was waived by the local ethics committee, as SEER data is publicly available and de-identified. The study was exempted from Institutional Review Board approval, due to the use of a large cancer database with unidentifiable patient information.

\section{References}

1. WHO I Suicide data. World Health Organization. 2018. Available online: http://www.who.int/mental_health/ prevention/suicide/suicideprevent/en/.

2. Misono S, Weiss NS, Fann JR, et al. Incidence of suicide in persons with cancer. J Clin Oncol 2008;26:4731-8.

3. Osazuwa-Peters N, Simpson MC, Zhao L, et al. Suicide risk among cancer survivors: Head and neck versus other cancers. Cancer 2018;124:4072-9.

4. Pham TT, Talukder AM, Walsh NJ, et al. Clinical and epidemiological factors associated with suicide in colorectal cancer. Support Care Cancer 2019;27:617-21.

5. Zhou H, Xian W, Zhang Y, et al. Trends in incidence and associated risk factors of suicide mortality in patients with non-small cell lung cancer. Cancer Med 2018;7:4146-55.

6. Simpson WG, Klaassen Z, Jen RP, et al. Analysis of Suicide Risk in Patients with Penile Cancer and Review of the Literature. Clin Genitourin Cancer 2018;16:e257-61.

7. Gaitanidis A, Alevizakos M, Pitiakoudis M, et al. Trends in incidence and associated risk factors of suicide mortality among breast cancer patients. Psychooncology 2018;27:1450-6.

8. AYA. 2015. Available online: https://www.cancer.gov/ types/aya.

9. Prevention AFoS. Suicide Statistics_-AFSP. Available online: https://afsp.org/about-suicide/suicide-statistics/. Accessed 9/8/2019.

10. Trama A, Botta L, Steliarova-Foucher E. Cancer Burden in Adolescents and Young Adults: A Review of Epidemiological Evidence. Cancer J 2018;24:256-66.

11. Bleyer A. Young Adult Oncology: The Patients and Their Survival Challenges. CA Cancer J Clin 2007;57:242-55.

12. About the SEER Program - SEER. Available online: https://seer.cancer.gov/about/overview.html.

13. Zhou H, Huang Y, Qiu Z, et al. Impact of prior cancer history on the overall survival of patients newly diagnosed with cancer: A pan-cancer analysis of the SEER database. Int J Cancer 2018;143:1569-77.

14. Urban D, Rao A, Bressel M, et al. Suicide in lung cancer: who is at risk? Chest 2013;144:1245-52.

15. Bass SB, Ruzek SB, Gordon TF, et al. Relationship of Internet health information use with patient behavior and self-efficacy: experiences of newly diagnosed cancer patients who contact the National Cancer Institute's Cancer Information Service. J Health Commun 2006;11:219-36.

16. Schiffman JD, Csongradi E, Suzuki LK. Internet use among adolescent and young adults (AYA) with cancer. Pediatr Blood Cancer 2008;51:410-5.

17. Penn A, Kuperberg A. Psychosocial Support in Adolescents and Young Adults With Cancer. Cancer J 2018;24:321-7.

18. Bradford A, Woodard TL. Novel Psychological Intervention for Decision Support in Women Considering Fertility Preservation Before Cancer Treatment. J Adolesc Young Adult Oncol 2017;6:348-52.

19. Allebeck P, Bolund C, Ringbäck G. Increased suicide rate in cancer patients. A cohort study based on the Swedish Cancer-Environment Register. J Clin Epidemiol 1989;42:611-6.

20. Rahouma M, Kamel M, Abouarab A, et al. Lung cancer patients have the highest malignancy-associated suicide rate in USA: a population-based analysis. Ecancermedicalscience 2018;12:859.

21. Teguh DN, Levendag PC, Noever I, et al. Treatment techniques and site considerations regarding dysphagiarelated quality of life in cancer of the oropharynx and nasopharynx. Int J Radiat Oncol Biol Phys 2008;72:1119-27.

22. Lahtinen S, Koivunen P, Ala-Kokko T, et al. Swallowingrelated quality of life after free flap surgery due to cancer of the head and neck. Eur Arch Otorhinolaryngol 


\section{Page 10 of 10}

2019;276:821-6.

23. McCarthy M. Suicide rates rise sharply in the US, figures show. BMJ 2016;353:i2355.

24. Hem E, Loge JH, Haldorsen T, et al. Suicide risk in cancer patients from 1960 to 1999. J Clin Oncol 2004;22:4209-16.

25. Siegel RL, Miller KD, Jemal A. Cancer statistics, 2018. CA Cancer J Clin 2018;68:7-30.

26. Xin Y, Huang Q, Tang JQ, et al. Nanoscale drug delivery for targeted chemotherapy. Cancer Lett 2016;379:24-31.

27. Darvin P, Toor SM, Sasidharan Nair V, et al. Immune checkpoint inhibitors: recent progress and potential biomarkers. Exp Mol Med 2018;50:165.

28. Fillon M. Immune checkpoint inhibitors are superior to docetaxel as second-line therapy for patients with non-small cell lung carcinoma. CA Cancer J Clin 2018;68:178-9.

29. Kendal WS. Suicide and cancer: a gender-comparative study. Ann Oncol 2007;18:381-7.

30. Parsons SK, Kumar AJ. Adolescent and young adult cancer care: Financial hardship and continued uncertainty. Pediatr Blood Cancer 2019;66:e27587.

31. Nock MK, Borges G, Bromet EJ, et al. Suicide and Suicidal Behavior. Epidemiol Rev 2008;30:133-54.

32. Assari S. Suicide Attempts in Michigan HealthCare System; Racial Differences. Brain Sci 2018;8. doi: 10.3390/ brainsci8070124.

33. Hirsch JK, Visser PL, Chang EC, et al. Race and ethnic differences in hope and hopelessness as moderators of the association between depressive symptoms and suicidal behavior. J Am Coll Health 2012;60:115-25.

34. Calati R, Ferrari C, Brittner M, et al. Suicidal thoughts and behaviors and social isolation: A narrative review of the literature. J Affect Disord 2019;245:653-67.

35. Hendifar AE, Petzel MQB, Zimmers TA, et al. Pancreas Cancer-Associated Weight Loss. Oncologist 2019;24:691-701.

36. Burnette CE, Roh S, Liddell J, et al. American Indian women cancer survivors' coping with depressive symptoms.

\section{Zhou et al. Suicide risk among AYA and all-age cancer survivors}

J Psychosoc Oncol 2018;37:494-508.

37. Ke Y, Ng T, Chan A. Survivorship care models for breast cancer, colorectal cancer, and adolescent and young adult (AYA) cancer survivors: a systematic review. Support Care Cancer 2018;26:2125-41.

38. Turaga KK, Malafa MP, Jacobsen PB, et al. Suicide in patients with pancreatic cancer. Cancer 2011;117:642-7.

39. Jayakrishnan TT, Sekigami Y, Rajeev R, et al. Morbidity of curative cancer surgery and suicide risk. Psychooncology 2017;26:1792-8.

40. Vyssoki B, Gleiss A, Rockett IR, et al. Suicide among 915,303 Austrian cancer patients: who is at risk? J Affect Disord 2015;175:287-91.

41. Pain management for patients with cancer. CA Cancer J Clin 2018;68:197-8.

42. Kjaer TK, Jensen A, Dalton SO, et al. Suicide in Danish women evaluated for fertility problems. Hum Reprod 2011;26:2401-7.

43. Kann L, McManus T, Harris WA, et al. Youth Risk Behavior Surveillance - United States, 2017. MMWR Surveill Summ 2018;67:1-114.

44. Moscicki EK, Umhau JC. Environmental Stressors May Drive Inflammation and Alter Neurocircuitry to Promote Suicidal Behavior. Curr Psychiatry Rep 2019;21:21.

45. Korhonen T, Sihvola E, Latvala A, et al. Early-onset tobacco use and suicide-related behavior - A prospective study from adolescence to young adulthood. Addict Behav 2018;79:32-8.

46. Sellers CM, Diaz-Valdes Iriarte A, Wyman Battalen A, et al. Alcohol and marijuana use as daily predictors of suicide ideation and attempts among adolescents prior to psychiatric hospitalization. Psychiatry Res 2019;273:672-7.

47. Clarke MC, Coughlan H, Harley M, et al. The impact of adolescent cannabis use, mood disorder and lack of education on attempted suicide in young adulthood. World Psychiatry 2014;13:322-3.

48. Coccia PF, Altman J, Bhatia S, et al. Adolescent and young adult oncology. Clinical practice guidelines in oncology. J Natl Compr Canc Netw 2012;10:1112-50.
Cite this article as: Zhou H, Xian W, Zhang Y, Yang Y, Fang W, Liu J, Shen J, Zhang Z, Hong S, Huang Y, Zhang L. Suicide among cancer patients: adolescents and young adult (AYA) versus all-age patients. Ann Transl Med 2019;7(22):658. doi: 10.21037/atm.2019.10.51 


\section{Supplementary}

Table S1 Suicide mortality rate in all-age patients and AYA patients, SEER, 1973-2015

\begin{tabular}{|c|c|c|c|c|c|c|c|}
\hline Cancer & All-age $^{a}$ & Suicide ${ }^{b}$ & SMR (\%) & $\mathrm{AYA}^{\mathrm{C}}$ & AYA suicide ${ }^{d}$ & AYA SMR (\%) & $\mathrm{P}^{\mathrm{e}}$ \\
\hline Nasopharynx & 12,146 & 41 & 0.34 & 2,131 & 8 & 0.38 & 0.783 \\
\hline Esophagus & 64,161 & 142 & 0.22 & 983 & 0 & 0.00 & 0.283 \\
\hline Stomach & 136,555 & 196 & 0.14 & 6,186 & 8 & 0.13 & 0.772 \\
\hline Liver & 107,008 & 60 & 0.06 & 2,920 & 1 & 0.03 & 0.621 \\
\hline Gallbladder & 19,787 & 14 & 0.07 & 322 & 0 & 0.00 & 1.000 \\
\hline Pancreas & 179,137 & 140 & 0.08 & 3,006 & 2 & 0.07 & 1.000 \\
\hline Lung & 863,239 & 1,142 & 0.13 & 10,548 & 12 & 0.11 & 0.603 \\
\hline Breast & 941,249 & 681 & 0.07 & 71,744 & 66 & 0.09 & 0.062 \\
\hline Cervix uteri & 76,452 & 75 & 0.10 & 25,166 & 39 & 0.15 & 0.019 \\
\hline Uterus & 206,425 & 114 & 0.06 & 9,715 & 8 & 0.08 & 0.271 \\
\hline Ovary & 111,708 & 84 & 0.08 & 10,246 & 12 & 0.12 & 0.148 \\
\hline Prostate & 972,409 & 2,553 & 0.26 & 1,038 & 3 & 0.29 & 1.000 \\
\hline Urinary bladder & 235,969 & 604 & 0.26 & 5,130 & 19 & 0.37 & 0.110 \\
\hline Kidney \& renal pelvis & 176,646 & 242 & 0.14 & 10,192 & 13 & 0.13 & 0.802 \\
\hline Eye & 15,750 & 33 & 0.21 & 1,523 & 3 & 0.20 & 1.000 \\
\hline
\end{tabular}

${ }^{\text {a }}$, number of all-age patients; ${ }^{b}$, number of suicide all-age patients; ${ }^{\mathrm{c}}$, number of AYA patients; ${ }^{\mathrm{d}}$, number of suicide AYA patients; ${ }^{\mathrm{e}}, \mathrm{P}$ value of chi-square test. SEER, Surveillance, Epidemiology, and End Results; AYA, adolescence and young adult; SMR, suicide mortality rate. 
Table S2 Trend of Suicide mortality SMR (\%) in all-age cancer patients, SEER, 1973-2015

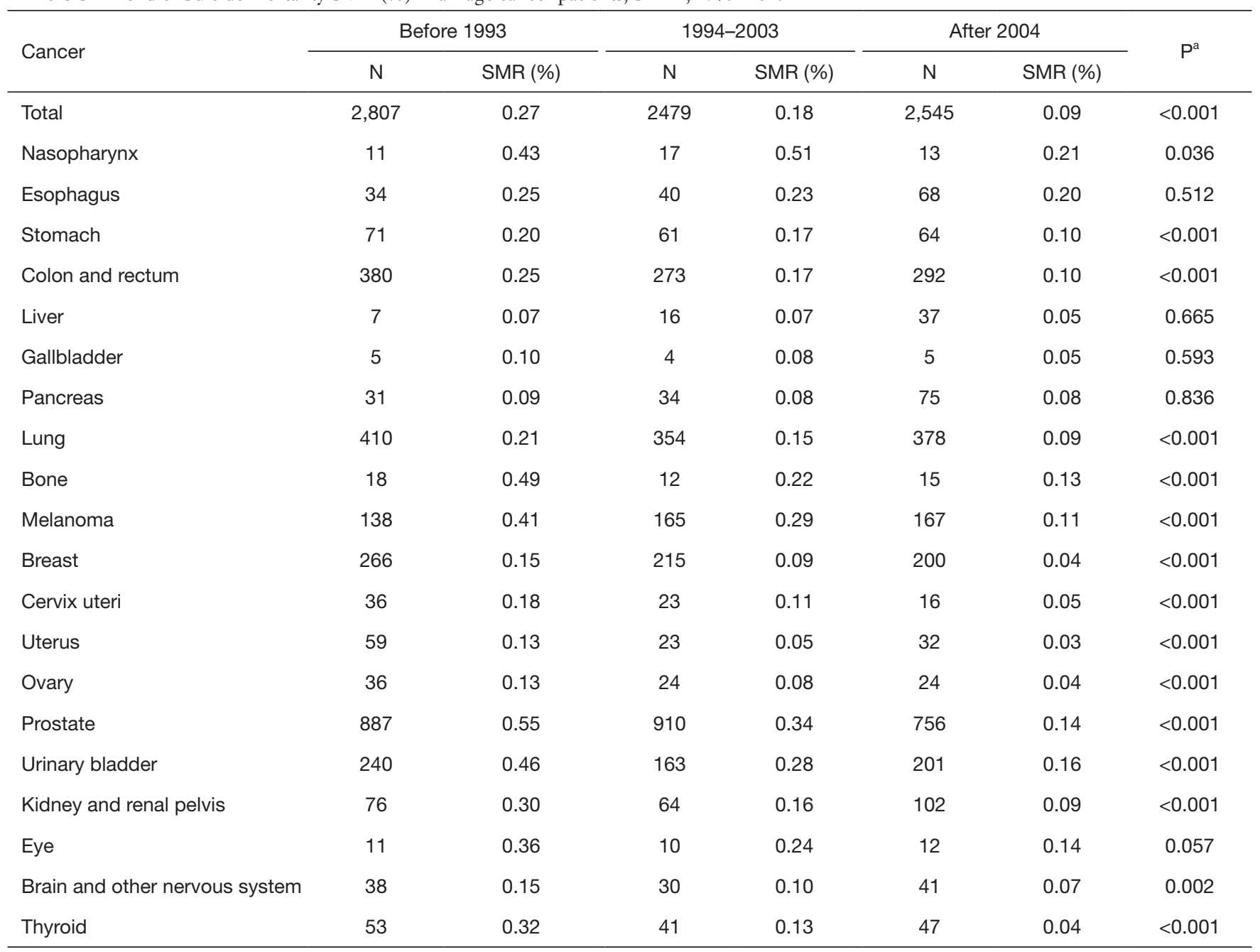

${ }^{\mathrm{a}}$, P value of chi-square test. SEER, Surveillance, Epidemiology, and End Results; SMR, suicide mortality rate. 
Table S3 Trend of suicide mortality SMR (\%) in AYA cancer patients, SEER, 1973-2015

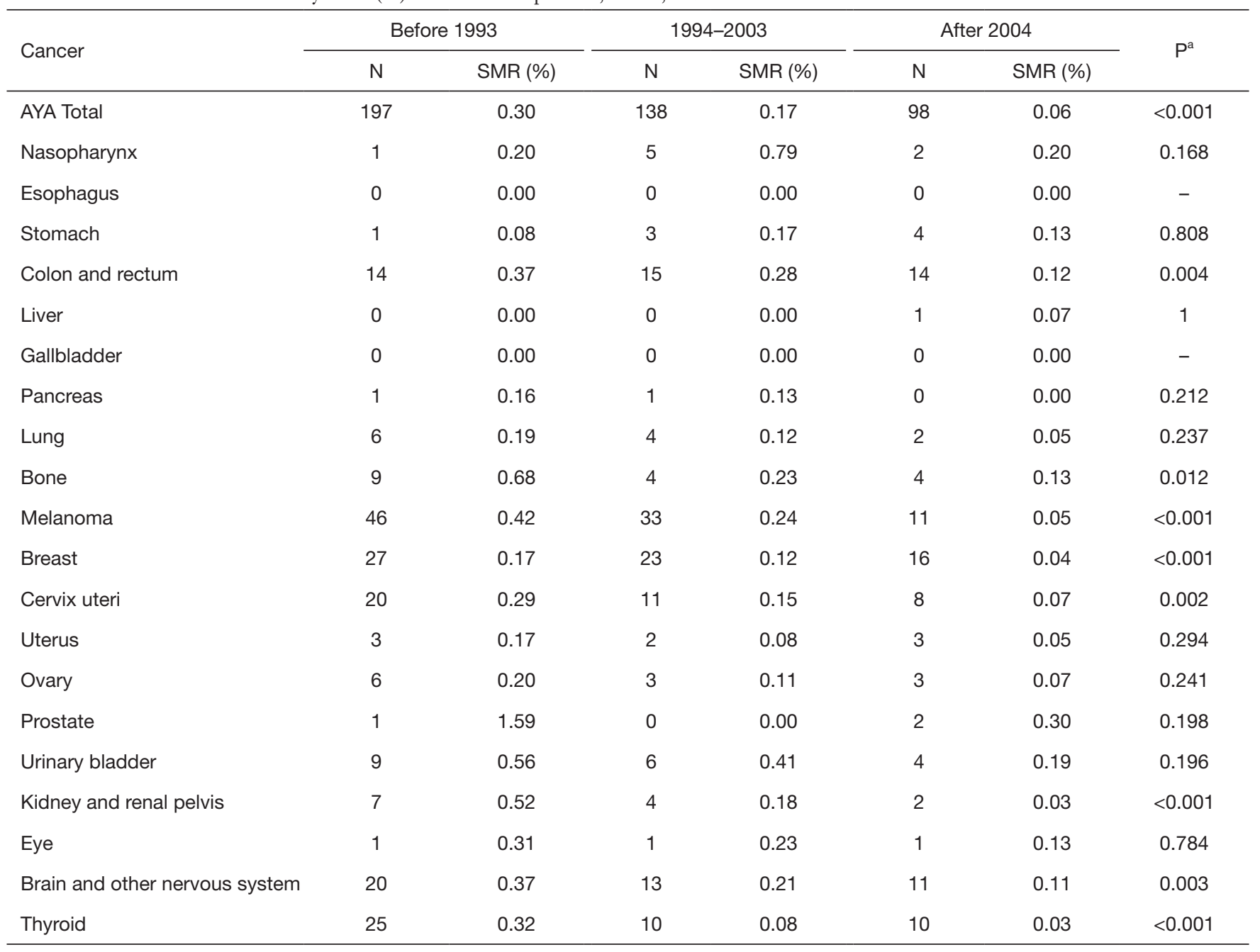

${ }^{\text {a }}$, P value of chi-square test. SEER, Surveillance, Epidemiology, and End Results; AYA, adolescence and young adult; SMR, suicide mortality rate. 